\title{
EFFECTS OF DIETARY COMPONENTS DURING AND AFTER CONCOMITANT CHEMORADIOTHERAPY, RADIOTHERAPY, OR SEQUENTIAL CHEMORADIOTHERAPY TO THE ABDOMINOPELVIC AREA
}

\author{
Laura Flores-Cisneros ${ }^{1,5, \S}$, Denisse Castro-Eguiluz $2, \S$, Diana Yolanda Reyes-Barretero ${ }^{3}$, \\ Emigdio Jaimes ${ }^{3}$, Claudia Cano-Blanco ${ }^{1}$, Carlos Avendaño-Pérez ${ }^{4}$, Berenice Carbajal-López ${ }^{1}$, \\ Miriam SÁnCheZ-López ${ }^{1}$, Aida Mota-García ${ }^{1}$, Dolores Gallardo-Rincón ${ }^{1}$, Marce InzUnza-SOto ${ }^{6}$, \\ Claudia Hernandez-Quintero ${ }^{6}$ and lucely Cetina-Pérez ${ }^{1 *}$ \\ ${ }^{1}$ Departments of Clinical Research,Medical Oncology, and Nutrition, Instituto Nacional de Cancerología, Mexico City; \\ ${ }^{2}$ Consejo Nacional de Ciencia y Tecnología (CONACyT) - Department of Clinical Research, Instituto Nacional de \\ Cancerología, Mexico City; ${ }^{3}$ Department of Nutrition, Centro Oncológico Estatal ISSEMyM, Toluca, Edo. de México, \\ Mexico; ${ }^{4}$ Department of Nutrition, Instituto Politécnico Nacional, Mexico City; ${ }^{5}$ Postgraduate Unit, Universidad \\ Nacional Autónoma de México, Mexico City, Mexico; ${ }^{6}$ Department of Nutrition, Universidad Autónoma de Sinaloa, \\ Academia de Ciencias de Nutrición y Gastronomía, Sinaloa, Sin., Mexico.
}

\begin{abstract}
Radiotherapy is a fundamental part of the treatment of pelvic neoplasms. Up to $90 \%$ of patients develop gastrointestinal symptoms as a result of acute injury to the small and large intestine, particularly in the mucosa. Radiotherapy leads to atrophy of the intestinal epithelium, acute crypt inflammation, inflammatory infiltration of the epithelium, malabsorption of lactose, and biliary salts as well as alterations in pancreatic enzymes and biliary salts, resulting in the malabsorption syndrome and dysbiosis. The most commonly reported symptoms of pelvic radiation disease include changes in bowel habits ( $94 \%)$, decreased fecal consistency (80\%), frequency of bowel movements (74\%), bowel urgency (39\%), and fecal incontinence (37\%). Although nutritional interventions with dietary modifications have been reported to prevent and treat gastrointestinal symptoms, the evidence remains inconclusive. (REV INVES CLIN. 2018;70:126-9)
\end{abstract}

Key words: Pelvic cancer. Radiotherapy. Chemotherapy. Diet. Fiber. Fat. Lactose. Gastrointestinal toxicity.

Corresponding author:

*Lucely Cetina-Pérez

Instituto Nacional de Cancerología

Av. San Fernando, No. 22

Col. Sección XVI, Del. Tlalpan

C.P. 14080, Ciudad de México, México

E-mail: lucelycetina.incan@gmail.com
$\S$ These authors contributed equally to this work.

Received for publication: 02-03-2018

Accepted for publication: 25-04-2018

doi: $10.24875 /$ RIC.18002525 


\section{INTRODUCTION}

Radiotherapy constitutes one of the cornerstones of treatment in cancer patients, comprising an average of 5-7 weeks of fractioned sessions. During therapy, $90 \%$ of patients develop gastrointestinal symptoms of variable severity due to the proximity of the gastrointestinal tract to the primary neoplasia ${ }^{1}$. The most common symptoms of pelvic radiation disease include changes in bowel habits (94\%), decreased fecal consistency (80\%), increased frequency of bowel movements (74\%), bowel urgency (39\%), and fecal incontinence $(37 \%)^{1}$.

The incidence and severity of chronic gastrointestinal symptoms are not completely related to the dosage of radiotherapy administered. They depend on the complex interaction between physical factors, treatment, and patient-related factors that remain poorly characterized. Reports suggest that between $6 \%$ and $78 \%$ of long-term cancer survivors' present gastrointestinal toxicity that affects their quality of life ${ }^{2}$.

Acute injury to the small and large intestine occurs mainly in the mucosa. Chronic injury is associated to damage on the intestinal wall ${ }^{3}$. Radiotherapy leads to atrophy of the intestinal epithelium, acute inflammation of the crypts, inflammatory infiltration of the epithelium, eosinophil infiltration, epithelium flattening, edema and collagen deposition, as well as alterations in pancreatic enzymes and biliary salts ${ }^{2}$. Malabsorption of lactose and biliary salts has also been observed during radiotherapy and may contribute to diarrhea. Non-hydrolyzed lactose in the intestinal lumen causes fluid accumulation, abdominal cramps, and watery diarrhea. High concentrations of biliary salts in the colon may interfere with the absorption and secretion of water and electrolytes, increasing bowel motility ${ }^{4}$. All these events lead to malabsorption syndrome and dysbiosis. Although different pathophysiological mechanisms may be involved in these events, they all lead to the development of pelvic radiation disease.

For the prevention and treatment of gastrointestinal symptoms caused by pelvic radiotherapy, nutritional interventions have been suggested, consisting of modifications in the intake of dietary fiber, fat, and lactose.

\section{Dietary fiber}

Dietary fiber is composed of complex carbohydrates, and it is classified as soluble or insoluble. Insoluble fiber acts as a bulking agent because it cannot be digested, while soluble fiber improves the integrity of feces due to its ability to absorb water ${ }^{5}$.

In a pilot study of patients undergoing pelvic radiotherapy, supplementation with psyllium hydrophilic mucilloid (5-20 ml/day), a hydrophilic soluble fiber concentrate that absorbs water in the intestine, decreased the incidence of diarrhea ( $60 \%$ in experimental vs. $83 \%$ in the control group, $p=0.049$ ). Furthermore, the group who received the mucilloid had a lower incidence of severe diarrhea (11 patients in experimental vs. 17 in the control group $)^{6}$. Level of evidence $B$, strength of recommendation 1 .

In a randomized clinical trial, to evaluate the longterm effect of nutritional intervention in patients treated with pelvic radiotherapy, patients who received the intervention were advised to decrease their dietary intake of insoluble fiber and lactose, while the control group remained on their usual diet ${ }^{7}$. The authors reported that the dietary intervention had no clear long-term effects on gastrointestinal symptoms or on the patients' quality of life.

However, they also mentioned that patients adhered poorly to the diet because no specific target was set in terms of the reduction in fiber and lactose intake. Furthermore, it was observed that the distinction between insoluble and soluble dietary fiber was complex for patients. The authors concluded that long-term gastrointestinal symptoms caused by radiotherapy were predominantly mild, and dietary advice suggesting the reduction of insoluble fiber and lactose intake was not superior to the maintenance of the usual diet in preventing the occurrence of these symptoms ${ }^{7}$. Level of evidence $A$, strength of recommendation 2 .

There is no evidence demonstrating a clear benefit of dietary fiber restriction in patients treated with pelvic radiotherapy; therefore, its restriction in these patients is not recommended. On the other hand, clinical trials evaluating the effect of a diet rich in soluble fiber are needed to assess whether these patients could benefit from it. 


\section{Fat}

As mentioned earlier, a high concentration of bile salts has been reported in the colon of patients undergoing pelvic radiotherapy ${ }^{4}$. This interferes with the absorption and secretion of water and electrolytes, thereby increasing intestinal motility. The use of cholestyramine to treat bile salt malabsorption has proven effective, but it may cause nausea and abdominal cramps. For this reason, several decades ago, the effect of a low-fat diet on the gastrointestinal radiation syndrome was studied ${ }^{8}$. In this study, bile salt malabsorption was measured through the excretion of C14 - cholic acid throughout radiotherapy and 2 years after treatment completion. Patients were placed on very low-fat diet ( $40 \mathrm{~g}$ ), and the excretion of bile salts decreased from $49 \%$ to $12 \%$ after 3-6 months on the diet $(p<0.01)$. Level of evidence $C$, strength of recommendation 2 .

However, more recent reports have described that bile salt malabsorption is an acute symptom which is only expressed during radiotherapy, tending to decrease over time after treatment completion. Hence, the effect of a very low-fat diet on bile salt excretion cannot be distinguished from its natural resolution over time, because the study did not include a control group following a usual $\operatorname{diet}^{8}$.

The effect of a diet low in fat and in lactose ( $40 \mathrm{~g}$ of fat and $5 \mathrm{~g}$ of lactose) to reduce the excretion of bile salts and the development of diarrhea has also been studied in comparison with a normal diet ( $80 \mathrm{~g}$ of fat) in patients undergoing pelvic radiotherapy ${ }^{4}$. Both diets appeared to decrease the incidence of diarrhea and the need for antidiarrheal medications during radiotherapy: 14 patients with a modified diet reported diarrhea versus 32 patients in the control group; the intervention group used 0.6 antidiarrheal tablets per day versus 1.1 in the control group. However, it is important to mention that although the group on the low-fat, low-lactose diet reported a decreased frequency of diarrhea, they reported a greater incidence of weight loss $>5 \%$, when compared with the control group ( $35 \%$ vs. $28 \%$ ). Therefore, based on this data, we cannot conclude that a low-fat, lowlactose diet is recommended in patients undergoing radiotherapy unless a positive effect from this modified diet is demonstrated on the patient's nutritional status and is not limited to the management of the gastrointestinal symptoms ${ }^{4}$. Level of evidence $B$, strength of recommendation 1 .

In some patients undergoing pelvic radiotherapy, the presence of steatorrhea has also been observed. It may be caused by pancreatic insufficiency, a sudden overgrowth of pathogenic bacteria, and malabsorption of bile salts. All are a consequence of radiotherapy, but steatorrhea will not be resolved until its underlying causes are addressed ${ }^{2}$.

The efficacy of a low- or modified-fat diet in the prevention of gastrointestinal toxicity was also studied in a randomized clinical trial ${ }^{9}$. A group of patients received a low-fat diet (20\% of total energy from longchain triglycerides) and another group received a diet modified in fat ( $20 \%$ of total energy from long-chain triglycerides plus $20 \%$ of total energy from mediumchain triglycerides); the control group followed a normal diet ( $40 \%$ of total energy from long-chain triglycerides). Changes in gastrointestinal toxicity were evaluated using the inflammatory bowel disease questionnaire-bowel (IBDQ-B). The mean (SE) decrease in paired IBDQ-B score was -7.3 (0.9) points, indicating a worsening toxicity; no significant differences were found between the three groups $(p=0.914$ between low-fat vs. modified-fat diet; $p=0.793$ between lowfat diet vs. control; and $p=0.89$ between modified-fat diet vs. control). The authors concluded that a low-fat or modified in fat diet during pelvic radiotherapy does not improve gastrointestinal symptoms in comparison with a normal diet. Nevertheless, it is important to note that there was an inadequate treatment adherence of fat intake among groups, and this may have interfered with the results. Level of evidence $B$, strength of recommendation 2 .

The evidence of a beneficial effect of a low-fat diet on the nutritional status of patients undergoing pelvic radiotherapy has not been sufficiently convincing.

\section{Lactose}

Lactose is a disaccharide found exclusively in dairy, and it is digested in the intestine by the enzyme lactase. Lactose intolerance is a syndrome caused by lactase deficiency, and it is characterized by abdominal cramps, diarrhea, nausea, and flatulence ${ }^{10}$. Lactase deficiency is present in $15 \%$ of patients undergoing pelvic radiotherapy due to the injury induced by 
radiation on the intestinal mucosa and the consequent decrease in brush border enzymes, including lactase, leading to lactose intolerance ${ }^{1}$.

Modification of lactose content in the diet has no effect on gastrointestinal toxicity in the long term ${ }^{7}$. When studying the effects of a diet low in lactose and fiber on gastrointestinal toxicity during pelvic radiotherapy, a tendency has been observed toward a lower prevalence of gastrointestinal symptoms. However, the quality of life was not different between groups, and gastrointestinal toxicity was not significantly different either ${ }^{7}$. Level of evidence $A$, strength of recommendation 1 .

A randomized clinical trial compared the effect of a diet modified in lactose in the prevention of radiation-induced diarrhea in patients treated with pelvic radiotherapy ${ }^{11}$. One group was assigned to a diet with lactose ( $480 \mathrm{ml}$ of whole milk), the second group to a diet with lactase ( $480 \mathrm{ml}$ of whole milk with lactase), and the third group received a lactose-restricted diet. The authors concluded that a lactose-restricted diet did not prevent radiation-induced diarrhea. Level of evidence A, strength of recommendation 1 .

As described earlier, evidence suggests that there is no beneficial effect of a low lactose diet in patients undergoing pelvic radiotherapy.

\section{CONCLUSION}

Due to the increased survival of cancer patients who have undergone radiotherapy treatment, it is essential to develop strategies to prevent gastrointestinal toxicity considering the particular characteristics of each patient. Nutritional intervention studies aimed to decrease these gastrointestinal effects are scarce and methodologically deficient. Hence, there is no evidence that dietary modification of fiber, fat, or lactose makes a difference and can improve gastrointestinal symptoms, nutritional status, and quality of life of patients receiving pelvic radiotherapy.

\section{REFERENCES}

1. Wedlake LJ, Shaw C, Whelan K, Andreyev HJ. Systematic review: the efficacy of nutritional interventions to counteract acute gastrointestinal toxicity during therapeutic pelvic radiotherapy. Aliment Pharmacol Ther. 2013;37:1046-56.

2. Andreyev J. Gastrointestinal complications of pelvic radiotherapy: are they of any importance? Gut. 2005;54:1051-4.

3. Coia LR, Myerson RJ, Tepper JE. Late effects of radiation therapy on the gastrointestinal tract. Int J Radiat Oncol Biol Phys. 1995:31:1213-36

4. Bye A, Kaasa S, Ose T, Sundfør K, Tropé C. The influence of low fat, low lactose. Clin Nutr 1992;11:147-53.

5. Elia M, Cummings JH. Physiological aspects of energy metabolism and gastrointestinal effects of carbohydrates. Eur J Clin Nutr. 2007;61 Suppl 1:S40-74.

6. Murphy J, Stacey D, Crook J, Thompson B, Panetta D. Testing control of radiation-induced diarrhea with a psyllium bulking agent: a pilot study. Can Oncol Nurs J. 2000;10:96-100.

7. Pettersson A, Nygren P, Persson C, et al. Effects of a dietary intervention on gastrointestinal symptoms after prostate cancer radiotherapy: long-term results from a randomized controlled trial. Radiother Oncol. 2014;113:240-7.

8. Bosaeus I, Andersson H, Nyström C. Effect of a low-fat diet on bile salt excretion and diarrhoea in the gastrointestinal radiation syndrome. Acta Radiol Oncol Radiat Phys Biol. 1979;18:460-4.

9. Wedlake LJ, McGough C, Shaw C, et al. Clinical trial: efficacy of a low or modified fat diet for the prevention of gastrointestinal toxicity in patients receiving radiotherapy treatment for pelvic malignancies. J Hum Nutr Diet. 2012;25:247-59.

10. Alliende F. Intolerancia a la lactosa y otros disacáridos. Gastr Latinoam. 2007;18:152-6.

11. Stryker JA, Bartholomew M. Failure of lactose-restricted diets to prevent radiation-induced diarrhea in patients undergoing whole pelvis irradiation. Int J Radiat Oncol Biol Phys. 1986; 12:789-92 